\title{
Multiple Attribute Decision-Making Model for Supplier Selection in Service-Oriented Manufacturing Paradigm
}

\author{
Feng Lyu $\mathbb{D}^{1},{ }^{1,2}$ Yanghang Zhang $\mathbb{D}^{1},{ }^{1}$ Zhuangzhuang Feng, ${ }^{1}$ Ruoyan Ding, ${ }^{3}$ and Jianxin Su ${ }^{1}$ \\ ${ }^{1}$ School of Mechatronics Engineering, Henan University of Science and Technology, Luoyang 471003, Henan, China \\ ${ }^{2}$ Collaborative Innovation Center of Machinery, Equipment Advanced Manufacturing of Henan Province, Luoyang 471003, \\ Henan, China \\ ${ }^{3}$ School of Management, Henan University of Science and Technology, Luoyang 471003, Henan, China \\ Correspondence should be addressed to Feng Lyu; lvfeng1980@haust.edu.cn
}

Received 19 January 2021; Revised 3 March 2021; Accepted 31 March 2021; Published 14 April 2021

Academic Editor: Tahir Mahmood

Copyright (c) 2021 Feng Lyu et al. This is an open access article distributed under the Creative Commons Attribution License, which permits unrestricted use, distribution, and reproduction in any medium, provided the original work is properly cited.

\begin{abstract}
This paper proposes a multiattribute decision-making model for supplier selection under the service-oriented manufacturing, which can be used to effectively evaluate each candidate supplier. The supplier selection index system under the service-oriented manufacturing is proposed, and the interval evaluation matrix is established. In view of the mixed attribute of evaluation index, we construct a method that converts mixed attribute value to interval number. In order to avoid the subjectivity of the weight and make alternatives be provided with more discrimination, we use a combination model based on the deviation function model and the interval relative entropy ranking method to evaluate each candidate supplier. Finally, an application example is given to verify the correctness and practicability of the proposed decision-making model.
\end{abstract}

\section{Introduction}

Service-oriented manufacturing is a new advanced manufacturing mode under the background of continuous integration, penetration, and enhancement of manufacturing and service; the advantages and importance of the SOM strategy are gradually recognized by more and more enterprises [1]. Service-oriented manufacturing is both manufacturing oriented by service and manufacturingbased service. Through the integration of products and services, full participation of customers, and mutual provision of productive services and service production by enterprises, the integration of decentralized manufacturing resources and high synergy of their core competitiveness can be achieved [2]. In the service-oriented manufacturing mode, in order to provide product service system to customers and meet personalized customer needs, enterprises in the value chain begin to focus more on their core capabilities and outsource their noncore business to other enterprises [3]. By integrating superior resources between enterprises and acquiring capabilities that cannot be provided by the enterprise itself, the overall value creation can be maximized through mutual cooperation, and the perceived value of customers can be maximized at the same time [4]. In this context, multiple enterprises with different core capabilities will form a community of interests or dynamic alliance, in which the supplier is an important node. As important components of the supply chain, suppliers usually play important roles in the manufacturing process [5]. Therefore, how to choose the right supplier and build an interactive and symbiotic network relationship with it becomes particularly important.

At present, scholars at home and abroad have made fruitful researches on supplier selection, mainly focusing on supplier evaluation criteria, supplier selection methods, and models. In the literatures of supplier selection, some works have been conducted on evaluation criteria and methodologies. Dickson was the first to make a systematic study on supplier evaluation criteria [6], to summarize the supplier evaluation criteria covering 23 indicators such as quality, cost, and delivery time. On this basis, Weber et al. studied the importance of evaluation 
criteria in supplier selection [7]. With the change of economic environment and the application of various advanced manufacturing modes, the supplier evaluation criteria are developing and improving continuously [8]. For example, complementary ability, synergy ability, and flexibility of suppliers begin to attract attention. Zhao et al. [9] hold that resource complementarity, cultural synergy, and prealliance linkage are the most important indicators for strategic alliance partner selection. Chen et al. [10] pointed out that the synergy of complementary resources and innovative resources is an important factor to create enterprise value. Guo et al. [11] pointed out that the service-oriented manufacturing makes fundamental changes in the relationship between enterprises and the way of value increment, and the supplier evaluation criteria should also be changed accordingly. Feng et al. [12] divided the service manufacturing network partner collaboration into two dimensions: complementary collaboration and interactive collaboration. Wang et al. [13] proposed that service-oriented manufacturing enterprises should pay more attention to suppliers' environmental performance, service capacity and quality, and cost flexibility when selecting suppliers. However, the existing evaluation criteria still have some shortcomings, which are reflected in the comprehensiveness of indicators that need to be improved, and most of the index values are accurate numbers. The research on the basis of traditional supply and demand relationship rather than strategic partnership is emphasized, and the new requirements for suppliers under the service-oriented manufacturing mode are not highly targeted.

Various methodologies utilized on supplier selection can be summarized as roughly intuitive judgment method, choice of open tender method, negotiation method, and other qualitative methods and cost method (purchasing cost comparison method and $\mathrm{ABC}$ cost method, etc.), the fuzzy theory (fuzzy clustering, intuitionistic fuzzy sets and fuzzy SMART, etc.), gray theory (gray relational analysis, etc.), multiple attribute decision-making (AHP and ANP, MAUT, outranking method, TOPSIS, etc.), multiple objective decision-making and mathematical programming (LP, GP, MIP, DEA, etc.), method of probability and statistics, artificial intelligence (evidence reasoning method, neural network, expert system, etc.), other methods (QFD, rough set theory, information entropy, VIKOR method, etc.), and integration method of these methods [14-16]. Zeng et al. [17] constructed a method based on the single-valued neutrosophic hybrid weighted similarity (SVNHWS) and entropy measures for handling SVN MADM problems. Wang et al. [18] studied some logarithmic distance measures and studied their usefulness in multiple attribute group decision-making (MAGDM) problems within single-valued neutrosophic linguistic (SVNL) environments. However, each method has its own advantages and disadvantages and needs to be improved [19]. For example, fuzzy theory has certain advantages in expressing expert opinions, but it usually needs to establish membership function or determine membership degree based on expert experience and judgment [20]. Multiobjective programming model can solve the conflicting objective problems in the process of supplier selection [21]. However, due to the complexity of specific problems, it is difficult to apply analytical methods to solve them.

With increasing complexity in the practical multiple attribute decision-making environment, decision-makers no longer are satisfied with using real numbers to represent their cognition for alternatives [22]. In view of this, this article is in reference to the basis of existing research results; according to the characteristics of the service-oriented manufacturing, service-oriented manufacturing mode is established under the supplier selection evaluation index system, constructs the decision model, and is proposed based on bias function model and the method of interval relative entropy sort, in order that service-oriented manufacturing enterprises effectively solve the vendor selection problem providing a reference to the ideas and methods.

\section{Establishment of Evaluation Criteria for Supplier Selection in Service-Oriented Manufacturing Mode}

Under the guidance of the scientific, comprehensive, and operational principles, combined with the characteristics of service-oriented manufacturing, and drawing on the research results of relevant scholars, the evaluation criteria for supplier selection in service-oriented manufacturing mode are designed. The factor layer consists of 7 parts: quality and technology, price, service level, synergy, flexibility, environmental performance, and comprehensive factors, each of which is refined by several indicators. See Table 1.

\subsection{Quality and Technical Factors}

(1) Product quality $B_{1}$ : it refers to the qualified rate of product delivery quality

(2) Quality assurance system $B_{2}$ : it refers to the completeness and effectiveness of the enterprise's quality assurance system, usually evaluated by experts, in the form of comments

(3) Technical level $B_{3}$ : it refers to the supplier's new product development ability, usually measured by the new product development rate

\subsection{Price Factors}

(1) Price $B_{4}$ : it refers to the price quoted by the supplier for the product/service. In order to be more competitive, the price usually takes the form of interval number.

(2) Cost saving ability $B_{5}$ : it refers to the cost saving ability of logistics on the premise of meeting the requirements of function and quality. Generally, it is expressed by the cost saving interval of average logistics cost per delivery. 
TABLE 1: Supplier evaluation criteria under service-oriented manufacturing mode.

\begin{tabular}{|c|c|c|}
\hline Target & Element & Indicators \\
\hline \multirow{24}{*}{ Supplier evaluation in service manufacturing mode } & & The quality of the product \\
\hline & Quality and technical factors & Quality assurance system \\
\hline & & The technical level \\
\hline & & The price \\
\hline & Price factors & Cost saving capability \\
\hline & & Service capability \\
\hline & & Order completion rate \\
\hline & Service-level factors & On-time delivery rate \\
\hline & & Delivery completion rate \\
\hline & & Complementary resources \\
\hline & & Collaborative innovation \\
\hline & Synergy factors & Interface management \\
\hline & & Cultural background \\
\hline & & The enterprise trust \\
\hline & & Quantity flexibility \\
\hline & The flexible factors & Variety flexibility \\
\hline & The flexible factors & Time flexibility \\
\hline & & Cost flexibility \\
\hline & Environmental performance & The effectiveness of environmental technologies \\
\hline & factors & Ecological efficiency \\
\hline & & The environmental costs \\
\hline & & Corporate reputation \\
\hline & Comprehensive factors & The management level \\
\hline & & Development potential \\
\hline
\end{tabular}

\subsection{Service-Level Factors}

(1) Service capacity $B_{6}$ : it refers to the elastic scale achieved by the supplier in terms of production and service quantity, which can be examined by the output within a certain period of time. This index is generally an interval value.

(2) Order completion rate $B_{7}$ : it reflects the supplier's ability to fulfill the contract, which can be expressed as the percentage of the actual completed orders in the planned completed orders.

(3) On-time delivery rate $\mathrm{B}_{8}$ : it refers to the ratio of the number of waybills delivered on time according to customer's requirements by the supplier within a certain period of time to the total amount of logistics within the same period.

(4) Delivery completion rate $\mathrm{B}_{9}$ : it refers to the proportion of goods delivered in good condition in a certain period of time to the total quantity.

\subsection{Synergy Factors}

(1) Complementary resources $B_{10}$ : it refers to the complementary strengths of suppliers and service manufacturing enterprises in human resource structure, core technology, knowledge management, capital, and other resources

(2) Collaborative innovation $B_{11}$ : this index reflects the scope and depth of innovation in the cooperation process in order to better respond to customer needs
(3) Interface management $B_{12}$ : it refers to the degree to which suppliers and service-oriented manufacturing enterprises, as well as different departments of enterprises, conduct interface docking and management through various information sharing platforms and technical means

(4) Cultural background $B_{13}$ : this index examines the attitude and willingness of both sides to be consistent and integrated in vision, goal, and cultural management

(5) Enterprise trust $B_{14}$ : it refers to the mutual trust between enterprises, which can be specifically investigated according to the depth, time, and performance of mutual cooperation

The above indicators can be assessed by experts in the form of comments.

\subsection{The Flexible Factors}

(1) Quantity flexibility $B_{15}$ : it refers to the supplier's ability to change the quantity of products within its production capacity

(2) Variety flexibility $B_{16}$ : it refers to the ability of suppliers to improve existing products and develop new products in order to adapt to changes in the market environment, as well as the ability to adjust varieties in the supply process

(3) Time flexibility $B_{17}$ : it refers to the ability of the supplier to shorten the delivery time in order to meet the customer demand 
(4) Cost flexibility $B_{18}$ : it refers to the enterprise's grasp of the cost structure at all stages of the product life cycle, reflecting the continuous competitive advantage of cost

The above indicators can be assessed by relevant experts in the form of grades and scores.

\subsection{Environmental Performance Factors}

(1) Effectiveness of environmental protection technology $\mathrm{B}_{19}$ : it refers to the effectiveness of the application of environmental protection technology by suppliers, which is usually evaluated by experts and given in the form of comments

(2) Eco-efficiency $B_{20}$ : it refers to the ratio of the value of products and services produced or provided by the supplier to the resources and energy consumed and the environmental load caused by them

(3) Environmental protection cost $\mathrm{B}_{21}$ : it refers to the cost of supplier's environmental protection input within a certain period of time

\subsection{Comprehensive Factors}

(1) Corporate reputation $\mathrm{B}_{22}$ : it refers to the reputation of the supplier in the industry

(2) Management level $B_{23}$ : it refers to the supplier's performance in internal system, organization, and management construction

(3) Development potential $B_{24}$ : it refers to the motivation and long-term planning of supplier's sustainable development

The above indicators can be assessed by relevant experts in the form of grades and scores.

\section{Decision Model Construction of Supplier Selection in Service-Oriented Manufacturing Mode}

Supplier selection in service-oriented manufacturing mode is a typical multilevel, uncertain, and multiattribute decision-making problem, which is usually transformed into a comparison and ranking problem of interval numbers [23].

In this research work, we define a set of suppliers to be evaluated as $P=\left\{p_{1}, p_{2}, \ldots, p_{s}, \ldots, p_{m}\right\}(s=1,2, \ldots, m)$, the indicator as $B=\left\{b_{1}, b_{2}, \ldots, b_{t}, \ldots, b_{n}\right\}(t=1,2, \ldots, n)$. The indexes are generally divided into two types: the higher the evaluation value, the better the efficiency index and the smaller the evaluation value, the better the cost index. Let the index weight vector $\omega=\left\{\omega_{1}, \omega_{2}, \ldots \omega_{n}\right\}$, and the size of $w$ represents the relative importance of each attribute index. $\omega_{t}$ is unknown, and $\sum_{t=1}^{n} \omega_{t}=1$.

3.1. Construction of Interval Evaluation Matrix. Let $c_{s t}$ represent the attribute value of the supplier $p_{s}$ under the evaluation index $b_{t}$ which can be obtained in various forms, such as exact number, interval number, and fuzzy number. These values form the initial evaluation matrix $C=\left(c_{s t}\right)_{m \times n}$.

The mixed initial matrix $C$ can be defined as

$$
C= \begin{cases}c_{s t} & \text { attribute value is the exact value, } \\ {\left[c_{s t}^{l}, c_{s t}^{u}\right],} & \text { attribute value is interval number, } \\ \left(c_{s t}^{l}, c_{s t}^{u}, c_{s t}^{r}\right), & \text { attribute value is fuzzy number. }\end{cases}
$$

In real life, the decision-makers prefer to utilize linguistic terms rather than employing the exact numbers owing to the complication of the socioeconomic environment and fuzziness of human beings thinking [24].

To make the experts' evaluation more accurate, a set of fuzzy linguistic values is set up and noted as \{Extremely Bad, Very Bad, Bad, Medium Bad, Medium, Medium Good, Good, Very Good, Extremely Good $\}$ ( $\{\mathrm{EB}, \mathrm{VB}, \mathrm{B}, \mathrm{MB}, \mathrm{M}$, MG, G, VG, EG\} for short). The evaluation data of qualitative criteria are given by experts in the form of fuzzy linguistic values that correspond to fuzzy numbers [25]. Mapping rules of linguistic variables and triangular fuzzy number are shown in Table 2.

In the process of supplier selection, the information of many indicators is not a definite number. In order for analysis and evaluation, the interval number is introduced to determine the attribute value of each index. Based on the generalization and operability of analysis and decision-making, the mixed attribute index is transformed into interval number index, and we construct the interval evaluation matrix $E$.

$$
E=\left|\left[e_{s t}^{l}, e_{s t}^{r}\right]\right|_{m \times n} .
$$

The original evaluation matrix $E$ was formulated as shown in

$$
\left[e_{s t}^{l}, e_{s t}^{r}\right]= \begin{cases}{\left[e_{s t}, e_{s t}\right],} & \text { attribute value is the exact value, } \\ {\left[e_{s t}^{l}, e_{s t}^{u}\right],} & \text { attribute value is interval number, } \\ {\left[\frac{\left(e_{s t}^{l}+e_{s t}^{u}\right)}{2}, \frac{\left(e_{s t}^{u}+e_{s t}^{r}\right)}{2}\right],} & \text { attribute value is riangular fuzzy number. }\end{cases}
$$


TABLE 2: Mapping rules of linguistic variables and triangular fuzzy numbers.

\begin{tabular}{lcc}
\hline No. & Linguistic evaluation value & Triangular fuzzy number \\
\hline 1 & Extremely bad (EB) & $(0.0,0.1,0.2)$ \\
2 & Very bad (VB) & $(0.1,0.2,0.3)$ \\
3 & Bad (B) & $(0.2,0.3,0.4)$ \\
4 & Medium bad (MB) & $(0.3,0.4,0.5)$ \\
5 & Medium (M) & $(0.4,0.5,0.6)$ \\
6 & Medium good (MG) & $(0.5,0.6,0.7)$ \\
7 & Good (G) & $(0.6,0.7,0.8)$ \\
8 & Very good (VG) & $(0.7,0.8,0.9)$ \\
9 & Extremely good (EG) & $(0.8,0.9,1.0)$ \\
\hline
\end{tabular}

3.2. Normalization of the Interval Number Matrix. In order to avoid the effect of adopting different units and to reduce the variability, the attribute values of the original indicators need to be normalized [26]. The normalized interval matrix $U$ can be determined by using (4). For a criterion if the larger value is better, it can be normalized by using (5), whereas for a criterion if the smaller value is better, it can be normalized by using (6).

$$
\begin{aligned}
& U=\left|\left[u_{s t}^{l}, u_{s t}^{r}\right]\right|_{m \times n}, \\
& \left\{\begin{array}{l}
u_{s t}^{l}=\frac{e_{s t}^{l}}{\sqrt{\sum_{s=1}^{m}\left(e_{s t}^{r}\right)^{2}}}, \\
u_{s t}^{r}=\frac{e_{s t}^{r}}{\sqrt{\sum_{s=1}^{m}\left(e_{s t}^{l}\right)^{2}}},
\end{array}\right. \\
& \left\{\begin{array}{l}
u_{s t}^{l}=\frac{\left(1 / e_{s t}^{r}\right)}{\sum_{s=1}^{m}\left(1 / e_{s t}^{l}\right)^{2}}, \\
u_{s t}^{r}=\frac{\left(1 / e_{s t}^{l}\right)}{\sum_{s=1}^{m}\left(1 / e_{s t}^{r}\right)^{2}} .
\end{array}\right.
\end{aligned}
$$

3.3. Determination of Criteria Weight. In this paper, in the evaluation process of some indicators, expert grading method is used to make quantitative evaluation. Its theoretical and systematic nature is still lacking; sometimes it is difficult to guarantee the objectivity and accuracy of the evaluation results. In order to reduce the subjective arbitrariness of the index weight, the deviation function model is used to determine the index weight, and the result obtained has scientific and mathematical theoretical basis and has strong objectivity.

Make $d\left(u_{s t}, u_{k t}\right)$ represent the distance between $u_{s t}$ and $u_{k t}$ in the normalized interval matrix $U$; then,

$$
d\left(u_{s t}, u_{k t}\right)=\frac{\sqrt{2}}{2} \sqrt{\left(u_{s t}^{l}-u_{k t}^{l}\right)^{2}+\left(u_{s t}^{r}-u_{k t}^{r}\right)^{2}} .
$$

For the indicator $b_{t}$, the deviation $D_{s t}(\omega)$ between candidate suppliers $p_{s}$ and other suppliers can be denoted as follows:

$$
D_{s t}(\omega)=\sum_{k=1}^{m} d\left(u_{s t}, u_{k t}\right) \omega_{t}, \quad(s=1,2, \ldots, m),(t=1,2, \ldots, n)
$$

The total deviation $D_{t}(\omega)$ between each candidate supplier and other suppliers can be denoted as follows:

$$
D_{t}(\omega)=\sum_{s=1}^{m} D_{s t}(\omega)=\sum_{s=1}^{m} \sum_{k=1}^{n} d\left(u_{s t}, u_{k t}\right) \omega_{t}, \quad(t=1,2, \ldots, n) .
$$

The selection of index weight vector should maximize the total deviation of all indexes to all candidate suppliers [27]. Therefore, the deviation function is established as follows:

$$
D(\omega)=\sum_{t=1}^{n} D_{t}(\omega)=\sum_{s=1}^{m} D_{s t}(\omega)=\sum_{s=1}^{m} \sum_{t=1}^{n} \sum_{k=1}^{m} d\left(u_{s t}, u_{k t}\right) \omega_{t}
$$

To maximize the deviation function, the Lagrange function is constructed as follows:

$$
L(\omega, \lambda)=\sum_{s=1}^{m} \sum_{t=1}^{n} \sum_{k=1}^{m} d\left(u_{s t}, u_{k t}\right) \omega_{t}+\frac{1}{2} \lambda\left[\sum_{t=1}^{n} \omega_{t}^{2}-1\right] .
$$

To find its partial derivative, make

$$
\left\{\frac{\partial L}{\partial \omega_{t}}=\sum_{s=1}^{m} \sum_{k=1}^{m} d\left(u_{s t}, u_{k t}\right)+\lambda \omega_{t}=0, \frac{\partial L}{\partial \lambda}=\sum_{t=1}^{n} \omega_{t}^{2}-1=0 .\right.
$$

To find the optimal solution of $\omega_{t}$ and normalize the weight vector, we can calculate the final solution:

$$
\omega_{t}=\frac{\sum_{s=1}^{m} \sum_{k=1}^{m} d\left(u_{s t}, u_{k t}\right)}{\sum_{t=1}^{n}\left[\sum_{s=1}^{m} \sum_{k=1}^{m} d\left(u_{s t}, u_{k t}\right)\right]}, \quad(t=1,2, \ldots, n) .
$$

3.4. Construction of Weighted Standardized Decision Matrix. The weighted standardized decision matrix $G$ is constructed by using

$$
G=\left[g_{s t}^{l}, g_{s t}^{r}\right]
$$

where

$$
\left\{\begin{array}{l}
g_{s t}^{l}=\omega_{t} \cdot u_{s t}^{l} \\
g_{s t}^{r}=\omega_{t} \cdot u_{s t}^{r}
\end{array}\right.
$$

3.5. Calculation of the Relative Entropy Distance between Each Candidate Supplier and Ideal Solution. The concept of relative entropy is used to calculate the relative entropy between each candidate supplier and ideal solution instead of the generalized distance which is commonly used in TOPSIS method. It solves the problem that TOPSIS method is unable to distinguish the point in perpendicular between the positive and negative ideal solutions [28]. 
The ideal scheme $\tilde{a}_{t}^{*}$ and negative ideal scheme $\tilde{a}_{t}^{-}$can be defined as follows:

$$
\begin{aligned}
& \tilde{a}_{t}^{*}=\max \left(\max a_{s t}^{l}, \max a_{s t}^{r}\right), \quad(t=1,2, \ldots, n), \\
& \tilde{a}_{t}^{-}=\min \left(\min a_{s t}^{l}, \min a_{s t}^{r}\right), \quad(t=1,2, \ldots, n) .
\end{aligned}
$$

Then, the ideal point of each attribute can be defined as follows:

$$
\begin{aligned}
& f_{t}^{*}=\left[\tilde{a}_{t}^{*}, \tilde{a}_{t}^{*}\right], \\
& f_{t}^{-}=\left[\widetilde{a}_{t}^{-}, \tilde{a}_{t}^{-}\right] .
\end{aligned}
$$

Set

$$
\tilde{g}_{t}=\left[g_{t}^{l}, g_{t}^{r}\right]
$$

For the ideal point,

$$
d\left(\tilde{g}_{t}, \tilde{f}_{t}^{*}\right)=d\left(g_{t}^{l}, a_{t}^{*}\right)+d\left(g_{t}^{r}, a_{t}^{*}\right),
$$

where

$$
\begin{aligned}
& d\left(g_{t}^{l}, a_{t}^{*}\right)=g_{t}^{l} \ln \frac{g_{t}^{l}}{a_{t}^{*}}+\left(1-g_{t}^{l}\right) \ln \frac{\left(1-g_{t}^{l}\right)}{\left(1-a_{t}^{*}\right)}, \\
& d\left(g_{t}^{r}, a_{t}^{*}\right)=g_{t}^{r} \ln \frac{g_{t}^{r}}{a_{t}^{*}}+\left(1-g_{t}^{*}\right) \ln \frac{\left(1-g_{t}^{r}\right)}{\left(1-a_{t}^{*}\right)} .
\end{aligned}
$$

Similarly, for the negative ideal point,

$$
d\left(\tilde{g}_{t}, \tilde{f}_{t}^{-}\right)=d\left(g_{t}^{l}, a_{t}^{-}\right)+d\left(g_{t}^{r}, a_{t}^{-}\right),
$$

where

$$
\begin{aligned}
& d\left(g_{t}^{r}, a_{t}^{-}\right)=g_{t}^{i} \ln \frac{g_{t}^{r}}{a_{t}^{-}}+\left(1-g_{t}^{-}\right) \ln \frac{\left(1-g_{t}^{r}\right)}{\left(1-a_{t}^{-}\right)}, \\
& d\left(g_{t}^{r}, a_{t}^{-}\right)=g_{t}^{i} \ln \frac{g_{t}^{r}}{a_{t}^{-}}+\left(1-g_{t}^{-}\right) \ln \frac{\left(1-g_{t}^{r}\right)}{\left(1-a_{t}^{-}\right)} .
\end{aligned}
$$

The relative entropy distance of each alternative $P_{s}$ from the ideal reference point and negative ideal reference point can be derived, respectively, as follows:

\begin{tabular}{|c|c|c|c|c|}
\hline Indicators & $P 1$ & $P 2$ & $P 3$ & $P 4$ \\
\hline $\mathrm{B}_{1}$ & 0.90 & 0.95 & 0.80 & 0.84 \\
\hline $\mathrm{B}_{2}$ & Good & Good & Ordinary & Nice \\
\hline $\mathrm{B}_{3}$ & 0.15 & 0.25 & 0.18 & 0.16 \\
\hline $\mathrm{B}_{4}$ & {$[270,275]$} & {$[275,290]$} & {$[260,266]$} & {$[265,269]$} \\
\hline $\mathrm{B}_{5}$ & {$[0.70,1.20]$} & {$[0.60,1.10]$} & {$[0.50,1.00]$} & {$[0.45,0.85]$} \\
\hline $\mathrm{B}_{6}$ & {$[300,310]$} & {$[320,330]$} & {$[280,290]$} & {$[300,310]$} \\
\hline $\mathrm{B}_{7}$ & 0.98 & 0.97 & 0.96 & 0.99 \\
\hline $\mathrm{B}_{8}$ & 0.94 & 0.97 & 0.90 & 0.92 \\
\hline $\mathrm{B}_{9}$ & 0.87 & 0.96 & 0.88 & 0.93 \\
\hline $\mathrm{B}_{10}$ & V-good & Good & Good & Ordinary \\
\hline $\mathrm{B}_{11}$ & V-good & Good & Nice & Nice \\
\hline $\mathrm{B}_{12}$ & Nice & Good & Ordinary & Nice \\
\hline $\mathrm{B}_{13}$ & Good & Nice & Nice & Nice \\
\hline $\mathrm{B}_{14}$ & Good & V-good & Good & Nice \\
\hline $\mathrm{B}_{15}$ & Nice & Good & Good & V-good \\
\hline $\mathrm{B}_{16}$ & Good & V-good & Good & Nice \\
\hline $\mathrm{B}_{17}$ & V-good & Nice & Nice & Nice \\
\hline $\mathrm{B}_{18}$ & Good & V-good & Good & Nice \\
\hline $\mathrm{B}_{19}$ & Nice & Good & Ordinary & Nice \\
\hline $\mathrm{B}_{20}$ & 0.9 & 1.3 & 0.7 & 0.8 \\
\hline $\mathrm{B}_{21}$ & 430 & 520 & 380 & 370 \\
\hline $\mathrm{B}_{22}$ & Good & V-good & Nice & Ordinary \\
\hline $\mathrm{B}_{23}$ & Good & Good & Nice & Nice \\
\hline $\mathrm{B}_{24}$ & Good & Good & Nice & Ordinary \\
\hline
\end{tabular}

$$
\begin{aligned}
& d_{s}^{*}=\sum_{t=1}^{n} d\left(\tilde{a}_{t}, \tilde{f}_{t}^{*}\right), \\
& d_{s}^{-}=\sum_{t=1}^{n} d\left(\tilde{a}_{t}, \tilde{f}_{t}^{*}\right) .
\end{aligned}
$$

3.6. Obtaining the Closeness Coefficient and Ranking the Order of Alternatives. The closeness coefficient between the candidate supplier and the ideal solution is calculated based on (28).

The relative closeness is introduced, and the calculation method is as follows:
TABLe 3: Candidate supplier initial decision matrix table.

An alternative $p_{i}$ with index $L_{s}^{*}$ approaching indicates that the alternative $p_{i}$ is close to the ideal reference point and far from the negative ideal reference point. Rank each $L_{s}^{*}$ of each alternative $p_{i}$ in descending order. The alternative $p_{i}$ with the highest $L_{s}^{*}$ value will be the best choice.

\section{An Illustrative Example}

To verify the feasibility and effectiveness of the proposed method, an illustrative example of supplier selection in service-oriented manufacturing paradigm is given in this paper.

The parts supplier of a service manufacturing enterprise often has problems such as delayed delivery and unguaranteed quality during the service process, so it is urgent to choose one of the four candidate parts suppliers after preliminary screening as its partner. Table 3 shows the historical data of the four suppliers and the initial decision values of the experts on the suppliers.

The interval decision matrix can be obtained by using (2) and (3), as shown in Table 4.

Except for attribute $\mathrm{B}_{4}$ which is cost type, other attributes are benefit type. The normalized decision matrix can be obtained by using (4)-(6). From (7) and (13), the attribute weight can be obtained by LINGO software. See Table 5.

Weighted normalized decision matrix can be obtained by using (14) and (15), as shown in Table 6.

According to (16)-(18), we can know the ideal points and negative ideal points of each attribute, as shown in Table 7. 
TABLE 4: Interval decision matrix table.

\begin{tabular}{|c|c|c|c|c|}
\hline & $P 1$ & $P 2$ & $P 3$ & $P 4$ \\
\hline $\mathrm{B}_{1}$ & {$[0.90,0.90]$} & {$[0.95,0.95]$} & {$[0.80,0.80]$} & {$[0.85,0.85]$} \\
\hline $\mathrm{B}_{2}$ & {$[0.65,0.75]$} & {$[0.65,0.75]$} & {$[0.45,0.55]$} & {$[0.55,0.65]$} \\
\hline $\mathrm{B}_{3}$ & {$[0.15,0.15]$} & {$[0.25,0.25]$} & {$[0.18,0.18]$} & {$[0.16,0.16]$} \\
\hline $\mathrm{B}_{4}$ & {$[270,275]$} & {$[275,290]$} & {$[260,266]$} & {$[265,269]$} \\
\hline $\mathrm{B}_{5}$ & {$[0.70,1.20]$} & {$[0.60,1.10]$} & {$[0.50,1.00]$} & {$[0.45,0.85]$} \\
\hline $\mathrm{B}_{6}$ & {$[300,310]$} & {$[320,330]$} & {$[280,290]$} & {$[300,310]$} \\
\hline $\mathrm{B}_{7}$ & {$[0.98,0.98]$} & {$[0.97,0.97]$} & {$[0.96,0.96]$} & {$[0.99,0.99]$} \\
\hline $\mathrm{B}_{8}$ & {$[0.94,0.94]$} & {$[0.97,0.97]$} & {$[0.90,0.90]$} & {$[0.92,0.92]$} \\
\hline $\mathrm{B}_{9}$ & {$[0.87,0.87]$} & {$[0.96,0.96]$} & {$[0.88,0.88]$} & {$[0.93,0.93]$} \\
\hline $\mathrm{B}_{10}$ & {$[0.75,0.85]$} & {$[0.65,0.75]$} & {$[0.65,0.75]$} & {$[0.45,0.55]$} \\
\hline $\mathrm{B}_{11}$ & {$[0.75,0.85]$} & {$[0.65,0.75]$} & {$[0.55,0.65]$} & {$[0.55,0.65]$} \\
\hline $\mathrm{B}_{12}$ & {$[0.55,0.65]$} & {$[0.65,0.75]$} & {$[0.45,0.55]$} & {$[0.55,0.65]$} \\
\hline $\mathrm{B}_{13}$ & {$[0.65,0.75]$} & {$[0.55,0.65]$} & {$[0.55,0.65]$} & {$[0.55,0.65]$} \\
\hline $\mathrm{B}_{14}$ & {$[0.65,0.75]$} & {$[0.75,0.85]$} & {$[0.65,0.75]$} & {$[0.55,0.65]$} \\
\hline $\mathrm{B}_{15}$ & {$[0.55,0.65]$} & {$[0.65,0.75]$} & {$[0.65,0.75]$} & {$[0.75,0.85]$} \\
\hline $\mathrm{B}_{16}$ & {$[0.65,0.75]$} & {$[0.75,0.85]$} & {$[0.65,0.75]$} & {$[0.55,0.65]$} \\
\hline $\mathrm{B}_{17}$ & {$[0.75,0.85]$} & {$[0.55,0.65]$} & {$[0.55,0.65]$} & {$[0.55,0.65]$} \\
\hline $\mathrm{B}_{18}$ & {$[0.65,0.75]$} & {$[0.75,0.85]$} & {$[0.65,0.75]$} & {$[0.55,0.65]$} \\
\hline $\mathrm{B}_{19}$ & {$[0.55,0.65]$} & {$[0.65,0.75]$} & {$[0.45,0.55]$} & {$[0.55,00.65]$} \\
\hline $\mathrm{B}_{20}$ & {$[0.90,0.90]$} & {$[1.30,1.30]$} & {$[0.70,0.70]$} & {$[0.80,0.80]$} \\
\hline $\mathrm{B}_{21}$ & {$[430,430]$} & {$[520,520]$} & {$[380,380]$} & {$[370,370]$} \\
\hline $\mathrm{B}_{22}$ & {$[0.65,0.75]$} & {$[0.75,0.85]$} & {$[0.55,0.65]$} & {$[0.45,0.55]$} \\
\hline $\mathrm{B}_{23}$ & {$[0.65,0.75]$} & {$[0.65,0.75]$} & {$[0.55,0.65]$} & {$[0.55,0.65]$} \\
\hline $\mathrm{B}_{24}$ & {$[0.75,0.85]$} & {$[0.65,0.75]$} & {$[0.55,0.65]$} & {$[0.45,0.55]$} \\
\hline
\end{tabular}

Table 5: Normalized decision matrix and index weights.

\begin{tabular}{|c|c|c|c|c|c|}
\hline & $P 1$ & $P 2$ & P3 & $P 4$ & Weight \\
\hline $\mathrm{B}_{1}$ & {$[0.5132,0.5132]$} & {$[0.5418,0.5418]$} & {$[0.4562,0.4562]$} & {$[0.4847,0.4847]$} & 0.0256 \\
\hline $\mathrm{B}_{2}$ & {$[0.4779,0.6455]$} & {$[0.4779,0.6455]$} & {$[0.3308,0.4734]$} & {$[0.4044,0.5594]$} & 0.0503 \\
\hline $\mathrm{B}_{3}$ & {$[0.3967,0.3967]$} & {$[0.6611,0.6611]$} & {$[0.4760,0.4760]$} & {$[0.4231,0.4231]$} & 0.0760 \\
\hline $\mathrm{B}_{4}$ & {$[0.4860,0.5084]$} & {$[0.4609,0.4992]$} & {$[0.5025,0.5280]$} & {$[0.4969,0.5180]$} & 0.0108 \\
\hline $\mathrm{B}_{5}$ & {$[0.3348,1.0515]$} & {$[0.2869,0.9638]$} & {$[0.2391,0.8762]$} & {$[0.2152,0.7448]$} & 0.0694 \\
\hline $\mathrm{B}_{6}$ & {$[0.4834,0.5161]$} & {$[0.5156,0.5494]$} & {$[0.4511,0.4828]$} & {$[0.4834,0.5161]$} & 0.0177 \\
\hline $\mathrm{B}_{7}$ & {$[0.5025,0.5025]$} & {$[0.4974,0.4974]$} & {$[0.4923,0.4923]$} & {$[0.5077,0.5077]$} & 0.0046 \\
\hline $\mathrm{B}_{8}$ & {$[0.5038,0.5038]$} & {$[0.5199,0.5199]$} & {$[0.4824,0.4824]$} & {$[0.4931,0.4931]$} & 0.0111 \\
\hline $\mathrm{B}_{9}$ & {$[0.4776,0.4776]$} & {$[0.5270,0.5270]$} & {$[0.4831,0.4831]$} & {$[0.5106,0.5106]$} & 0.0158 \\
\hline $\mathrm{B}_{10}$ & {$[0.5115,0.6699]$} & {$[0.4433,0.5911]$} & {$[0.4433,0.5911]$} & {$[0.3069,0.4335]$} & 0.0596 \\
\hline $\mathrm{B}_{11}$ & {$[0.5139,0.6741]$} & {$[0.4454,0.5948]$} & {$[0.3769,0.5155]$} & {$[0.3769,0.5155]$} & 0.0466 \\
\hline $\mathrm{B}_{12}$ & {$[0.4206,0.5861]$} & {$[0.4971,0.6763]$} & {$[0.3441,0.4959]$} & {$[0.4206,0.5861]$} & 0.0451 \\
\hline $\mathrm{B}_{13}$ & {$[0.4805,0.6503]$} & {$[0.4066,0.5636]$} & {$[0.4066,0.5636]$} & {$[0.4066,0.5636]$} & 0.0217 \\
\hline $\mathrm{B}_{14}$ & {$[0.4314,0.5735]$} & {$[0.4978,0.6500]$} & {$[0.4314,0.5735]$} & {$[0.3650,0.4971]$} & 0.0386 \\
\hline $\mathrm{B}_{15}$ & {$[0.3650,0.4971]$} & {$[0.4314,0.5735]$} & {$[0.4314,0.5735]$} & {$[0.4978,0.6500]$} & 0.0386 \\
\hline $\mathrm{B}_{16}$ & {$[0.4170,0.4631]$} & {$[0.4811,0.6056]$} & {$[0.4170,0.5344]$} & {$[0.4811,0.6056]$} & 0.0365 \\
\hline $\mathrm{B}_{17}$ & {$[0.5317,0.7011]$} & {$[0.3899,0.5361]$} & {$[0.3899,0.5361]$} & {$[0.3899,0.5361]$} & 0.0415 \\
\hline $\mathrm{B}_{18}$ & {$[0.4314,0.5735]$} & {$[0.4978,0.6500]$} & {$[0.4314,0.5735]$} & {$[0.3650,0.4971]$} & 0.0386 \\
\hline $\mathrm{B}_{19}$ & {$[0.4206,0.5861]$} & {$[0.4971,0.6763]$} & {$[0.3441,0.4959]$} & {$[0.4206,0.5861]$} & 0.0451 \\
\hline $\mathrm{B}_{20}$ & {$[0.4724,0.4724]$} & {$[0.6823,0.6823]$} & {$[0.3674,0.3674]$} & {$[0.4199,0.4199]$} & 0.0896 \\
\hline $\mathrm{B}_{21}$ & {$[0.5010,0.5010]$} & {$[0.6059,0.6059]$} & {$[0.4428,0.4428]$} & {$[0.4311,0.4311]$} & 0.0523 \\
\hline $\mathrm{B}_{22}$ & {$[0.4585,0.6144]$} & {$[0.5290,0.6963]$} & {$[0.3879,0.5325]$} & {$[0.3174,0.4506]$} & 0.0687 \\
\hline $\mathrm{B}_{23}$ & {$[0.4631,0.6228]$} & {$[0.4631,0.6228]$} & {$[0.3919,0.5398]$} & {$[0.3919,0.5398]$} & 0.0278 \\
\hline $\mathrm{B}_{24}$ & {$[0.5290,0.6963]$} & {$[0.4585,0.6144]$} & {$[0.3879,0.5325]$} & {$[0.3174,0.4506]$} & 0.0687 \\
\hline
\end{tabular}

In order to sort candidate suppliers, interval relative entropy method is applied to obtain the relative entropy distance between candidate suppliers and ideal scheme and negative ideal scheme according to (19)-(27). The relative closeness between candidate suppliers and ideal solutions can be obtained according to (28), and then the advantages and disadvantages of candidate suppliers can be ranked, as shown in Table 8.

Therefore, the rank order of the suppliers is $P_{2}>P_{1}>P_{3}>P_{4}$. 
TABLE 6: Table of weighted normalized decision matrix.

\begin{tabular}{|c|c|c|c|c|}
\hline & $P 1$ & $P 2$ & $P 3$ & $P 4$ \\
\hline $\mathrm{B}_{1}$ & {$[0.0132,0.0132]$} & {$[0.0139,0.0139]$} & {$[0.0117,0.0117]$} & {$[0.0124,0.0124]$} \\
\hline $\mathrm{B}_{2}$ & {$[0.0241,0.0325]$} & {$[0.0241,0.0325]$} & {$[0.0166,0.0238]$} & {$[0.0204,0.0282]$} \\
\hline $\mathrm{B}_{3}$ & {$[0.0301,0.0301]$} & {$[0.0502,0.0502]$} & {$[0.0362,0.0362]$} & {$[0.0322,0.0322]$} \\
\hline $\mathrm{B}_{4}$ & {$[0.0052,0.0055]$} & {$[0.0050,0.0054]$} & {$[0.0054,0.0057]$} & {$[0.0054,0.0056]$} \\
\hline $\mathrm{B}_{5}$ & {$[0.0232,0.0730]$} & {$[0.0199,0.0669]$} & {$[0.0166,0.0608]$} & {$[0.0149,0.0517]$} \\
\hline $\mathrm{B}_{6}$ & {$[0.0085,0.0091]$} & {$[0.0091,0.0097]$} & {$[0.0080,0.0085]$} & {$[0.0085,0.0091]$} \\
\hline $\mathrm{B}_{7}$ & {$[0.0023,0.0023]$} & {$[0.0023,0.0023]$} & {$[0.0023,0.0023]$} & {$[0.0023,0.0023]$} \\
\hline $\mathrm{B}_{8}$ & {$[0.0056,0.0056]$} & {$[0.0058,0.0058]$} & {$[0.0053,0.0053]$} & {$[0.0055,0.0055]$} \\
\hline $\mathrm{B}_{9}$ & {$[0.0075,0.0075]$} & {$[0.0083,0.0083]$} & {$[0.0076,0.0076]$} & {$[0.0081,0.0081]$} \\
\hline $\mathrm{B}_{10}$ & {$[0.0305,0.0399]$} & {$[0.0264,0.0352]$} & {$[0.0264,0.0352]$} & {$[0.0183,0.0258]$} \\
\hline $\mathrm{B}_{11}$ & {$[0.0239,0.0314]$} & {$[0.0208,0.0277]$} & {$[0.0176,0.0240]$} & {$[0.0176,0.0240]$} \\
\hline $\mathrm{B}_{12}$ & {$[0.0190,0.0264]$} & {$[0.0224,0.0305]$} & {$[0.0155,0.0224]$} & {$[0.0190,0.0264]$} \\
\hline $\mathrm{B}_{13}$ & {$[0.0104,0.0141]$} & {$[0.0088,0.0122]$} & {$[0.0088,0.0122]$} & {$[0.0088,0.0122]$} \\
\hline $\mathrm{B}_{14}$ & {$[0.0166,0.0221]$} & {$[0.0192,0.0251]$} & {$[0.0166,0.0221]$} & {$[0.0141,0.0192]$} \\
\hline $\mathrm{B}_{15}$ & {$[0.0141,0.0192]$} & {$[0.0166,0.0221]$} & {$[0.0166,0.0221]$} & {$[0.0192,0.0251]$} \\
\hline $\mathrm{B}_{16}$ & {$[0.0152,0.0169]$} & {$[0.0176,0.0221]$} & {$[0.0152,0.0195]$} & {$[0.0176,0.0221]$} \\
\hline $\mathrm{B}_{17}$ & {$[0.0220,0.0291]$} & {$[0.0162,0.0222]$} & {$[0.0162,0.0222]$} & {$[0.0162,0.0222]$} \\
\hline $\mathrm{B}_{18}$ & {$[0.0166,0.0221]$} & {$[0.0192,0.0251]$} & {$[0.0166,0.0221]$} & {$[0.0141,0.0192]$} \\
\hline $\mathrm{B}_{19}$ & {$[0.0190,0.0264]$} & {$[0.0224,0.0305]$} & {$[0.0155,0.0224]$} & {$[0.0190,0.0264]$} \\
\hline $\mathrm{B}_{20}$ & {$[0.0423,0.0423]$} & {$[0.0611,0.0611]$} & {$[0.0329,0.0329]$} & {$[0.0376,0.0376]$} \\
\hline $\mathrm{B}_{21}$ & {$[0.0262,0.0262]$} & {$[0.0317,0.0317]$} & {$[0.0232,0.0232]$} & {$[0.0226,0.0226]$} \\
\hline $\mathrm{B}_{22}$ & {$[0.0315,0.0422]$} & {$[0.0363,0.0478]$} & {$[0.0266,0.0366]$} & {$[0.0218,0.0309]$} \\
\hline $\mathrm{B}_{23}$ & {$[0.0129,0.0173]$} & {$[0.0129,0.0173]$} & {$[0.0109,0.0150]$} & {$[0.0109,0.0150]$} \\
\hline $\mathrm{B}_{24}$ & {$[0.0363,0.0478]$} & {$[0.0315,0.0422]$} & {$[0.0266,0.0366]$} & {$[0.0218,0.0309]$} \\
\hline
\end{tabular}

TABLE 7: Ideal and negative ideal points for attributes.

\begin{tabular}{lcc}
\hline Indicators & Ideal point & Negative ideal point \\
\hline $\mathrm{B}_{1}$ & {$[0.0139,0.0139]$} & {$[0.0117,0.0117]$} \\
$\mathrm{B}_{2}$ & {$[0.0325,0.0325]$} & {$[0.0166,0.0166]$} \\
$\mathrm{B}_{3}$ & {$[0.0502,0.0502]$} & {$[0.0301,0.0301]$} \\
$\mathrm{B}_{4}$ & {$[0.0057,0.0057]$} & {$[0.0050,0.0050]$} \\
$\mathrm{B}_{5}$ & {$[0.0730,0.0730]$} & {$[0.0150,0.0150]$} \\
$\mathrm{B}_{6}$ & {$[0.0097,0.0097]$} & {$[0.0080,0.0080]$} \\
$\mathrm{B}_{7}$ & {$[0.0023,0.0023]$} & {$[0.0023,0.0023]$} \\
$\mathrm{B}_{8}$ & {$[0.0058,0.0058]$} & {$[0.0053,0.0053]$} \\
$\mathrm{B}_{9}$ & {$[0.0083,0.0083]$} & {$[0.0075,0.0075]$} \\
$\mathrm{B}_{10}$ & {$[0.0399,0.0399]$} & {$[0.0183,0.0183]$} \\
$\mathrm{B}_{11}$ & {$[0.0314,0.0314]$} & {$[0.0176,0.0176]$} \\
$\mathrm{B}_{12}$ & {$[0.0305,0.0305]$} & {$[0.0155,0.0155]$} \\
$\mathrm{B}_{13}$ & {$[0.0141,0.0141]$} & {$[0.0088,0.0088]$} \\
$\mathrm{B}_{14}$ & {$[0.0251,0.0251]$} & {$[0.0141,0.0141]$} \\
$\mathrm{B}_{15}$ & {$[0.0251,0.0251]$} & {$[0.0141,0.0141]$} \\
$\mathrm{B}_{16}$ & {$[0.0221,0.0221]$} & {$[0.0152,0.0152]$} \\
$\mathrm{B}_{17}$ & {$[0.0291,0.0291]$} & {$[0.0162,0.0162]$} \\
$\mathrm{B}_{18}$ & {$[0.0251,0.0251]$} & {$[0.0141,0.0141]$} \\
$\mathrm{B}_{19}$ & {$[0.0305,0.0305]$} & {$[0.0155,0.0155]$} \\
$\mathrm{B}_{20}$ & {$[0.0611,0.0611]$} & {$[0.0329,0.0329]$} \\
$\mathrm{B}_{21}$ & {$[0.0317,0.0317]$} & {$[0.0226,0.0226]$} \\
$\mathrm{B}_{22}$ & {$[0.0478,0.0478]$} & {$[0.0218,0.0218]$} \\
$\mathrm{B}_{23}$ & {$[0.0173,0.0173]$} & {$[0.0109,0.0109]$} \\
$\mathrm{B}_{24}$ & {$[0.0478,0.0478]$} & {$[0.0218,0.0218]$} \\
\hline
\end{tabular}

TABLE 8: Relative entropy distance and relative closeness from ideal scheme and negative ideal scheme.

\begin{tabular}{|c|c|c|c|c|}
\hline Candidate supplier & Ideal scheme relative entropy distance & Negative ideal scheme relative entropy distance & Relative closeness & Sorting \\
\hline $\mathrm{P}_{1}$ & 0.0683 & 0.1379 & 0.6688 & 2 \\
\hline $\mathrm{P}_{2}$ & 0.0535 & 0.1603 & 0.7498 & 1 \\
\hline $\mathrm{P}_{3}$ & 0.1134 & 0.0741 & 0.3952 & 3 \\
\hline $\mathrm{P}_{4}$ & 0.1276 & 0.0560 & 0.3050 & 4 \\
\hline
\end{tabular}


The priority of candidate suppliers is $P_{2}>P_{1}>P_{3}>P_{4} . P_{2}$ should be selected as the partner of this service manufacturing enterprise, which is consistent with the supplier actually determined.

\section{Conclusions}

This paper constructs a reasonable supplier evaluation index system in service-oriented manufacturing, which includes quality and technology, price, service-level, collaborative ability, flexibility, environmental performance, and comprehensive factors according to the new characteristics of supplier selection in service-oriented manufacturing; the index system improves the evaluation criteria of supplier selection, transforms the language evaluation value into the form of triangular fuzzy number, and gives each index value in the form of interval number. The combined model based on the deviation function model and the interval relative entropy ranking method is used to evaluate the candidate suppliers. The deviation function model is used to determine the weight of the attribute, which can better avoid the subjectivity problem. The interval relative entropy sorting method is used to make the scheme sorting more discriminative and improve the accuracy of decision-making. By establishing a scientific and reasonable supplier selection system, the supply network of service-oriented manufacturing enterprises can be optimized to maximize the overall value creation with suppliers, realize the integration of manufacturing resources in the supply chain, improve the overall management level of the enterprise, and enhance the core competence of the enterprise. An application example shows the effectiveness and practicability of the proposed method, which provides a new method to select the best supplier for service-oriented manufacturing enterprises and also provides new ideas for other multiattribute decision-making problems.

\section{Data Availability}

The data used to support the findings of this study are available from the corresponding author.

\section{Conflicts of Interest}

The authors declare that they have no conflicts of interest.

\section{Acknowledgments}

This work was supported by the National Key R\&D Program of China (2020YFB1713500), Innovation Method Fund of China (2016IM030200), Key Scientific Research Projects of Higher Education Institutions in Henan Province (20B410002), and Doctoral Scientific Research Foundation of Henan University of Science and Technology.

\section{References}

[1] Z. Lu, "An analytical study on service-oriented manufacturing strategies," International Journal of Production Economics, vol. 139, no. 1, pp. 220-228, 2012.
[2] J. Gao, Y. Yao, V. C. Y. Zhu, L. Sun, and L. Lin, "Service-oriented manufacturing: a new product pattern and manufacturing paradigm," Journal of Intelligent Manufacturing, vol. 22, no. 3, pp. 435-446, Jun. 2011.

[3] W. Lin, Z. Jiang, and N. Li, "A survey on the research of service-oriented manufacturing," Industrial Engineering Management, vol. 14, no. 6, pp. 1-31, 2009.

[4] J. Chen, S. Tong, and S. Yao, "Research on value co-creation mechanism in service manufacturing," Science and Technology Progress and Policy, vol. 31, no. 1, pp. 435-446, 2014.

[5] Q. Zhang, Z. Guo, F. Man, and J. Ma, "Evaluation and selection of manufacturing suppliers in B2B E-commerce environment," Complexity, vol. 2020, pp. 1-8, 2020.

[6] G. W. Dickson, "AN analysis OF vendor selection systems and decisions," Journal of Purchasing, vol. 2, no. 1, pp. 5-17, 1966.

[7] C. A. Weber, J. R. Current, and W. C. Benton, "Vendor selection criteria and methods," European Journal of Operational Research, vol. 50, no. 1, pp. 2-18, 2007.

[8] Y. Yuan, T. Guan, X. Yan, and Y. Li, "Based on hybrid VIKOR method decision making model for supplier selection," Control and Decision, vol. 29, no. 3, pp. 551-560, 2014.

[9] C. Zhao and Y. Jiang, "Empirical research on Chinese enterprise strategic alliance partners characters matching standard," Studies in Science of Science, vol. 28, no. 4, pp. 558-565, 2010.

[10] S. Chen, X. Shi, and S. Wu, "Impact of complementary assets and innovation asset synergy on performance-from the Prospect of moderating effect of environmental dynamism," Systems Engineering, vol. 33, no. 1, pp. 61-67, 2015.

[11] R. Guo and Z. He, "FNN Based vendor selection in serviceoriented manufacturing enterprises," Journal of Xi'an Technological University, vol. 31, no. 4, pp. 340-344, 2011.

[12] B. Feng, W. Suo, and Z. Fan, "Evaluation on collaborative performances of a service-manufacturing network considering the fuzzy correlations of multicriteria," Chinese Journal of Management Science, vol. 20, no. 4, pp. 95-103, 2012.

[13] L. Wang, L. Sun, and T. Feng, "Study on vendor selection in service-oriented manufacturing enterprises," Commercial Research, vol. 23, no. 2, pp. 1-5, 2010.

[14] A. Sanayei, S. Farid Mousavi, and A. Yazdankhah, "Group decision making process for supplier selection with VIKOR under fuzzy environment," Expert Systems with Applications, vol. 37, no. 1, pp. 24-30, 2010.

[15] W. Ho, X. Xu, and P. K. Dey, "Multi-criteria decision making approaches for supplier evaluation and selection: a literature review," European Journal of Operational Research, vol. 202, no. 1, pp. 16-24, 2010.

[16] J. Chai, J. N. K. Liu, and E. W. T. Ngai, "Application of decision-making techniques in supplier selection: a systematic review of literature," Expert Systems with Applications, vol. 40, no. 10, pp. 3872-3885, 2013.

[17] S. Zeng, Y. Hu, T. Balezentis, and D. Streimikiene, "A multicriteria sustainable supplier selection framework based on neutrosophic fuzzy data and entropy weighting," Sustainable Development, vol. 28, no. 5, pp. 1431-1440, 2020.

[18] J. Wang, S. Zeng, and C. Zhang, "Single-valued neutrosophic linguistic logarithmic weighted distance measures and their application to supplier selection of fresh aquatic products," Mathematics, vol. 8, no. 3, p. 14, 2020.

[19] B. Zheng and F. Liu, "The determination of weights in supplier selection by using DEA/TOPSIS model," Industrial Engineering Journal, vol. 06, pp. 55-59, 2011.

[20] L. Y. Zhai, L. P. Khoo, and Z. W. Zhong, "Towards a QFDbased expert system: a novel extension to fuzzy QFD 
methodology using rough set theory," Expert Systems with Applications, vol. 37, no. 12, pp. 8888-8896, 2010.

[21] X. Liu, H. Li, C. Wang, and C. Chu, "A survey of supplier selection models and approaches," Chinese Journal of Management Science, vol. 12, no. 1, pp. 139-148, 2004.

[22] X. Deng, J. Wang, and G. Wei, "Multiple attribute decision making based on power muirhead mean operators under 2tuple linguistic pythagorean fuzzy environment," Cognitive Computation, vol. 2020, 2020.

[23] H. Zhao, Y. Bao, and S. Guan, "Study on sorting method of interval numbers in multiple attribute decision making and its application," Mathematics in Practice and Theory, vol. 43, no. 19 , pp. 43-48, 2013.

[24] F. Lei, G. Wei, H. Gao, J. Wu, and C. Wei, "TOPSIS method for developing supplier selection with probabilistic linguistic information," International Journal of Fuzzy Systems, vol. 22, no. 3, pp. 749-759, 2020.

[25] Y. Feng, Z. Zhang, G. Tian et al., "A novel hybrid fuzzy grey TOPSIS method: supplier evaluation of a collaborative manufacturing enterprise," Applied Sciences-Basel, vol. 9, no. 18, p. 25, 2019.

[26] P. Pitchipoo, P. Venkumar, and S. Rajakarunakaran, "Grey decision model for supplier evaluation and selection in process industry: a comparative perspective," International Journal of Advanced Manufacturing Technology, vol. 76, no. 9-12, pp. 2059-2069, 2015.

[27] W. Tang, D. Ding, and S. Liu, "Research on supplier selection based on mix-attribute index," Journal of Hefei University of Technology, vol. 34, no. 3, pp. 457-461, 2011.

[28] Y. Su, X. Meng, Z. Zhao, and Z. Li, "Cognitive virtual network embedding algorithm based on weighted relative entropy," Ksii Transactions on Internet and Information Systems, vol. 13, no. 4, pp. 1845-1865, 2019. 